\title{
REPRESENTATION THEOREMS FOR UNCONDITIONALLY CONVERGING OPERATORS
}

\author{
JOE HOWARD AND KENNETH MELENDEZ
}

ABSTRACT. Let $N(X)$ be the set $\left\{F \in X^{\prime \prime}\right.$ : there exists a weakly unconditionally converging series $\Sigma x_{\eta}$ in $X$ such that $F=\sigma\left(X^{\prime \prime}, X^{\prime}\right)$ $\left.\lim _{n} \sum_{i=1}^{n} J x_{i}\right\}$. Representation theorems for the unconditionally converging operators (map weakly unconditionally converging series into unconditionally converging series) are developed by using the $\sigma\left(X^{\prime}, N(X)\right)$ topology of $X^{\prime}$.

Let $T$ be a continuous linear operator which maps a Banach space $X$ into a Banach space $Y . T$ is said to be unconditionally converging (uc) if $T$ maps weakly unconditionally converging (wuc) series into unconditionally converging (uc) series. A series $\Sigma x_{i}$ in $X$ is uc if for each subseries $\sum x_{k_{i}}$, there is an element $x \in X$ such that $x=\sigma\left(X, X^{\prime}\right)-\lim _{n} \sum_{i=1}^{n} x_{k_{i}}$; and is wuc if $\Sigma\left|f\left(x_{i}\right)\right|<\infty$ for every $f \in X^{\prime} . N(X)$ is to denote the set $\left\{F \in X^{\prime \prime}\right.$ : there exists a wuc series $\Sigma x_{n}$ in $X$ such that $\left.F=\sigma\left(X^{\prime \prime}, X^{\prime}\right)-\lim _{n} \sum_{i=1}^{n} J x_{i}\right\}_{0}$ It is clear that $N(X)$ is a linear subspace of $X^{\prime \prime}$ containing $J X$ ( $J$ is the natural map of $X$ into $\left.X^{\prime \prime}\right)$. Note that $\sigma\left(X^{\prime}, N(X)\right)$ is a topology on $X^{\prime}$ weaker than (or equal to) the weak topology and stronger than (or equal to) the weak ${ }^{*}$ topology. A subset $B^{\prime}$ of $X^{\prime}$ is said to be wuc-limited if $\lim _{n} \sup _{B^{\prime}} x^{\prime}\left(x_{n}\right)$ $=0$ for every wuc series $\Sigma x_{n}$ in $X$.

Operator representation theorems are given for compact and weakly compact operators in [2] and [6]. We develop similar representation theorems for the unconditionally converging operators by using the $\sigma\left(X^{\prime}, N(X)\right)$ topology.

1. Characterizing a uc operator. Let $T^{\prime}$ and $T^{\prime \prime}$ denote respectively the first and second adjoint of $T$. The uc operator can be characterized as follows.

Theorem 1.1. Let $T: X \rightarrow Y$. The following are equivalent.

(a) $T$ is uc.

Received by the editors October 12, 1972.

AMS (MOS) subject classifications (1970). Primary 46G10.

Key words and phrases. Operator representations, unconditionally converging operator, unconditionally converging series. 
(b) $T^{\prime}$ maps bounded sets of $Y^{\prime}$ into wuc-limited sets of $X^{\prime}$.

Proof. (a) $\Rightarrow$ (b): Let $T$ be uc and $\Sigma x_{n}$ be wuc. Then $\Sigma T x_{n}$ is uc. Hence, $T x_{n} \rightarrow 0$, and we obtain

Therefore, $T{ }^{\prime} B$ ' is wuc-limited.

$$
0=\lim _{n} \sup _{B^{\prime}} y^{\prime}\left(T x_{n}\right)=\lim _{n} \sup _{T^{\prime} B^{\prime}} T^{\prime} y^{\prime}\left(x_{n}\right) .
$$

(b) $\Rightarrow$ (a): Now suppose $\Sigma x_{n}$ is wuc. Let $U^{\prime}$ be the unit disk of $X^{\prime}$. Then

$$
0=\lim _{n} \sup _{T^{\prime} U^{\prime}} T^{\prime} y^{\prime}\left(x_{n}\right)=\lim _{n} \sup _{U^{\prime}} y^{\prime}\left(T x_{n}\right)=\lim _{n}\left\|T x_{n}\right\| .
$$

Hence, $\inf _{n}\left\|T x_{n}\right\|=0$, so by $\left[1\right.$, Theorem 5], $\Sigma T x_{n}$ is uc.

Corollary 1.2. Let $B^{\prime}$ be a bounded set in $X^{\prime}$. Then $B^{\prime}$ is $\sigma\left(X^{\prime}, N(X)\right)$ conditionally compact if and only if $B^{\prime}$ is a wuc-limited set.

Proof. Define $S=\left\{x \in X:\left|b^{\prime}(x)\right| \leq 1\right.$ for every $\left.b^{\prime} \in B^{\prime}\right\}$. Then $S$ is a closed subset in $X$ by [7, p. 238]. If $p$ is the gauge of $S$, then $p^{\prime}$ is a norm on $X / \operatorname{Ker} p$, where $p^{\prime}(\hat{x})=p(x), x=\hat{x}+\operatorname{Ker} p$. Let $T$ be the natural map from $X$ to $Y=X / \operatorname{Ker} p$. If $U^{\prime}$ is the unit disk of $Y^{\prime}$, then $T^{\prime} U^{\prime}=B^{\prime}$. Now $B^{\prime}$ is $\sigma\left(X^{\prime}, N(X)\right)$ conditionally compact $\Leftrightarrow T$ is uc $\Leftrightarrow T^{\prime}$ maps $U^{\prime}$ onto a wuc-limited set $\Leftrightarrow B^{\prime}$ is a wuc-limited set.

2. Representation theorems for uc operators. Let $S$ be a compact Hausdorff space and let $T$ be a continuous linear operator from $X$ to $C(S)$. From $[2, \mathrm{p} .490]$ we know there exists a mapping $v: S \rightarrow X^{\prime}$ which is continuous with the $\sigma\left(X^{\prime}, X\right)$ topology such that:

(a) $T x(s)=v(s) x$ for $x \in X$ and $s \in S$;

(b) $\|T\|=\sup _{s \in S}|v(s)|$.

Conversely, if given such a map $v$, then the operator $T$ defined by (a) is a continuous linear operator from $X$ to $C(S)$ with norm given by (b). Here $v$ is defined by $v=T^{\prime} \pi$ where $\pi: S \rightarrow C(S)$ is given by $\pi(s) f=f(s), f \in C(S)$, $s \in S . \pi$ is a homeomorphism of $S$ into a compact subset of $C^{\prime}(S)$ with the $\sigma\left(C^{\prime}(S), C(S)\right)$ topology.

Theorem 2.1. $T$ is uc if and only if $v$ is $\sigma\left(X^{\prime}, N(X)\right)$ continuous.

Proof. If $T$ is uc, then $T^{\prime}$ is $\sigma\left(C^{\prime}(S), C(S)\right)-\sigma\left(X^{\prime}, N(X)\right)$ continuous; thus $v=T^{\prime} \pi$ will be $\sigma\left(X^{\prime}, N(X)\right)$ continuous.

Conversely, suppose $v$ is $\sigma\left(X^{\prime}, N(X)\right)$ continuous. Let $\Sigma y_{n}$ be a wuc series in $X$ and $\Sigma x_{n}$ be an arbitrary subseries of $\Sigma y_{n}$. It suffices to show that $B=\left\{\sum_{i=1}^{n} T x_{i} \mid n=1,2, \cdots\right\}$ is $\sigma\left(C(S), C^{\prime}(S)\right)$ conditionally compact, 
since then $T$ would be uc (i.e $\Sigma T y_{n}$ would be $\sigma\left(C(S), C^{\prime}(S)\right.$ ) subseries convergent, hence uc). $B \subseteq C(S)$, so by $[2$, Theorem $14, \mathrm{p} .269] B$ is $\sigma\left(C(S), C^{\prime}(S)\right)$ conditionally compact if and only if $B$ is bounded and quasi-equicontinuous But $B=T A$ where $A=\left\{\sum_{i=1}^{n} x_{i} \mid n=1,2, \cdots\right\}$, and $A$ is bounded, so $B$ is bounded.

To see that $B$ is quasi-equicontinuous, let $s_{a} \rightarrow s$ in $S$, let $\epsilon>0$, and let $a_{0}$ be given. Since $v$ is $\sigma\left(X^{\prime}, N(X)\right)$ continuous, $v\left(s_{\alpha}\right) \rightarrow v(s)$ in $\sigma\left(X^{\prime}, N(X)\right)$. Since $\Sigma x_{n}$ is wuc, $\sum_{i=1}^{n} J x_{i} \rightarrow_{n}^{w^{*}} F \in N(X)$. Thus, $A_{1}=A \cup\{F\}$ is a $\sigma\left(X^{\prime \prime}, X^{\prime}\right)$ compact set and $v\left(s_{a}\right)$ and $v(s)$ are in $C\left(A_{1}\right)$. Since $v(s) \epsilon$ $C\left(A_{1}\right)$, then by Arzela's theorem [2, Theorem 11, p. 268] the convergence $v\left(s_{\alpha}\right) \rightarrow v(s)$ is quasi-uniform on $A_{1}$, hence, quasi-uniform on $A$. Thus, there exists a finite set of indices $\alpha_{1}, \ldots, \alpha_{n} \geq \alpha_{0}$ such that for each $a \in A$

$$
\begin{gathered}
\min _{1 \leq i \leq n}\left|v\left(s_{a_{i}}\right) a-v(s) a\right|<\epsilon ; \\
\min _{1 \leq i \leq n}\left|v\left(s_{a_{i}}\right) \sum_{j=1}^{k} x_{j}-v(s) \sum_{j=1}^{k} x_{j}\right|<\epsilon \text { for each } k ; \\
\min _{1 \leq i \leq n}\left|\sum_{j=1}^{k} T x_{j}\left(s_{a_{i}}\right)-\sum_{j=1}^{k} T x_{j}(s)\right|<\epsilon \text { for each } k ; \\
\min _{1 \leq i \leq n}\left|f\left(s_{a_{i}}\right)-f(s)\right|<\epsilon \text { for each } f \in B .
\end{gathered}
$$

Thus, $B$ is quasi-equicontinuous, and it therefore follows that $B$ is $\sigma\left(C(S), C^{\prime}(S)\right)$ conditionally compact, and hence $T$ is uc.

Let $S$ be an arbitrary topological space and $B(S)$ the space of bounded scalar-valued functions on $S$ with the sup norm. Then the operator representation theorem is appreciably simpler to prove.

Theorem 2.2. Let $T: X \rightarrow B(S)$ and $p: S \rightarrow X^{\prime}$ be such that $p$ is a bounded map with $T x(s)=p(s) x$. Then $T$ is uc if and only if $p(S)$ is $\sigma\left(X^{\prime}, N(X)\right)$ conditionally compact.

Proof. By Corollary 1.2, it suffices to prove the theorem for $p(S)$ a wuclimited set.

Assume $T$ is uc and let $\Sigma x_{n}$ be a wuc series. Then $\Sigma T x_{n}$ is a uc series; hence, $T x_{n} \rightarrow 0$, so

$$
\lim _{n} \sup _{p(s)} p(s) x=\lim _{n} \sup _{s} T x_{n}(s)=0 .
$$

Therefore, $p(S)$ is a wuc-limited set. 
Conversely, let $p(S)$ be any bounded subset of $X^{\prime}$ and assume $p(S)$ is wuc-limited. Let $\Sigma x_{n}$ be a wuc series. Then

$$
\lim _{n} \sup _{S} T x_{n}(s)=\lim _{n} \sup _{p(S)} p(s) x_{n}=0 .
$$

Hence, inf ${ }_{n}\left\|T x_{n}\right\|=0$, so by [1, Theorem 5], $\Sigma T x_{n}$ is uc.

Denote the bounded operators from $X$ to $Y$ by $B(X, Y)$ and the uc operators by $U C(X, Y)$.

Corollary 2.3. The following conditions are equivalent.

(a) $B(X, B(S))=U C(X, B(S))$.

(b) $U^{\prime}$ (the unit disk of $X^{\prime}$ ) is $\sigma\left(X^{\prime}, N(X)\right.$ ) compact.

(c) $U^{\prime}$ is wuc-limited.

Proof. This follows from Theorem 2.2 and Corollary 1.2.

\section{REF ERENCES}

1. C. Bessaga and A. Pel'czyński, On bases and unconditional convergence of series in Banach spaces, Studia Math. 17 (1958), 151-164. MR 22 \#5872.

2. N. Dunford and J. T. Schwartz, Linear operators. I: General theory, Pure and Appl. Math., vol. 7, Interscience, New York, 1958. MR 22 \#8302.

3. J. Howard, The comparison of an unconditionally converging operator, Studia Math. 33 (1969), 295-298. MR 40 \#785.

4. J. Howard and K. Melendez, Characterizing operators by their first and second adjoints (to appear).

5. C. W. McArthur, A note on subseries convergence, Proc. Amer. Math. Soc. 12 (1961), 540-545. MR 23 \#A3443.

6. E. D. Thorp and R. J. Whitley, Operator representation theorems, Illinois J. Math. 9 (1965), 595-601. MR 31 \#6126.

7. A. Wilansky, Functional analysis, Blaisdell, New York, 1964. MR 30 \#425.

DEPARTMENT OF MATHEMATICS AND STATISTICS, OKL AHOMA STATE UNIVERSITY, STILLWATER, OKLAHOMA 74074

Current address (Joe Howard): Route 2, Box 324, Portales, New Mexico 88130

Current address (Kenneth Melendez): U. S. Air Force, Hanscom Field, Bedford, Massachusetts 01730 